Orthopäde 2014 · 43:148-155

DOI 10.1007/s00132-013-2183-0

Online publiziert: 24. November 2013

(c) The Author(s) 2013. This article is published

with open access at link.springer.com
H. Schläfer · M. Schläfer

Orthopädisch-Unfallchirurgische Abteilung, St.-Elisabeth-Krankenhaus, Rodalben

\section{Achs- und rotationsgerechte Positionierung des bikondylären Femurschlittens}

\section{Hintergrund und Fragestellung}

Eine gerade mechanische Beinachse [9], eine adäquate Rotation der Prothesenteile $[2,4,5,10,11,13-16]$ und eine harmonische Balancierung der Weichteile in Extension und Flexion sind für das klinische Ergebnis nach endoprothetischer Versorgung mit einem kompletten Oberflächenersatz des Kniegelenks maßgeblich $[6,9,10]$. Die praktische Umsetzung macht die gegenseitige Beeinflussung dieser Parameter augenscheinlich und kann den Operateur zu Kompromissen zwingen $[3,17]$. Die operativen Vorgehensweisen der Operateure sind auch heute noch sehr unterschiedlich, führt man sich die Femur-first-, Tibia-first-, Streckspaltfirst-, Flexionsspalt-first-Techniken vor Augen. Sie stehen für Unterschiede in der Reihenfolge der Operationsschritte und/ oder in der Gewichtung von Ligamentbalance bzw. Landmarkorientierung. Die Bedeutung einer geraden mechanischen Beinachse und damit einer Positionierung des Femurschlittens im rechten Winkel zur mechanischen Femurachse ist schon lange unbestritten [9]. Eine direkte Definition der mechanischen $\mathrm{Fe}$ murachse ist intraoperativ unsicher. Auch ihre indirekte Bestimmung über die anatomische Femurachse und den Alphawinkel ist unsicher, da eine eindeutige Definition der anatomischen Femurachse nicht existiert, insbesondere bei gebogenen Femurschäften.
Die Rotation der Prothesenteile ist als dritte Dimension bei deren Implantation Ende der 90er Jahre Anfang 2000 in den Mittelpunkt des Interesses gerückt $[2,4,5$, $10,11,13,14,15]$. Diesbezüglich haben sich folgende entscheidende Erkenntnisse herauskristallisiert:

Die Transepikondylarachse (EKA) kommt der transversalen Drehachse des Kniegelenks am nächsten [8], diese Achse ist allerdings intraoperativ nicht sicher definierbar [12].

Die anatomischen Formen der Kniegelenke können erheblich voneinander abweichen ( $\bullet$ Abb. 1a). So kann der hintere Kondylenwinkel (Winkel zwischen EKA und hinterer Femurkondylentangente) erheblich schwanken [15]. Nach eigenen Untersuchungen liegt die Schwankungsbreite zwischen 0 und $13^{\circ}(\bullet$ Abb. 1b).

Eine standardisierte Ausrichtung des Femurschlittens in $3^{\circ}$ Außenrotation zur hinteren Kondylentangente ist deshalb nicht zielführend, da dadurch eine Vielzahl von Femurschlitten in Innenrotation implantiert wird.

Die Rotation der Femurprothese hat einen erheblichen Einfluss auf den femoropatellaren Gleitvorgang und die Balancierung des Flexionsspalts $[3,11,14,16]$. Dagegen können leichte Fehlrotationen der Tibiaprothese durch mobile Polyethylen-Inlays (PEs) ausgeglichen werden [7].

Die bisherigen Erkenntnisse führen zu den Postulaten, dass der bikondyläre Femurschlitten im rechten Winkel zur mechanischen Femurachse und parallel zur
EKA implantiert werden sollte, solange die Ligamentbalance dies zulässt.

Zur möglichst genauen Prothesenpositionierung werden auch heute noch ganz unterschiedliche Wege beschritten, die von der „Freihandtechnik“ ohne wesentliche präoperative Planung " unter Verwendung der prothesenspezifischen Schablonen, die Anwendung der computergestützten Navigation und/oder eingehender klinischer und röntgenologischer präoperativer Analyse bis hin zur Nutzung MRT- oder CT-gestützter individueller Operationsschablonen reichen. Die „Freihandtechnik“ ist unbestritten sehr unsicher. Mit Hilfe der Navigation ist die EKA nicht sicherer zu definieren als in der Freihandtechnik. Eine gerade mechanische Beinachse ist aber sichergestellt. Mit Abstrichen trifft dies auch für eine gute präoperative klinische und röntgenologische Planung zu.

Durch die Verwendung individueller Operationsschablonen ist eine korrekte Prothesenimplantation gewährleistet. Die weichteilige Balancierung allerdings erfolgt in der Regel sekundär, sodass die Rückzugsmöglichkeiten im Sinne eines knöchernen Kompromisses eingeschränkt sind. Auch werden die Kosten für individuelle Operationsschablonen von den Gesetzlichen Krankenkassen nur in Ausnahmefällen und von einem Teil der Privaten Krankenkassen getragen.

Diese Situation hat uns veranlasst, eine eigene Vorgehensweise zu entwickeln, die 
sicher und preiswert ist, Patienten nur geringfügig mit Röntgenstrahlen belastet und die Operationszeit zudem deutlich reduziert.

Diese Methode wurde vom Erstautor persönlich entwickelt.

\section{Material und Methode}

Seit 5 Jahren wird diese selbst entwickelte Vorgehensweise in unserer Abteilung angewendet. Seither wurden 783 bikondyläre Schlittenprothesen unter Anwendung dieser Methode als Standardmethode implantiert.

\section{Ziel der Methode}

Das Ziel unserer Methode ist, den bikondylären Schlitten im rechten Winkel zur mechanischen Femurachse (MF) und parallel zur Transepikondylarachse (EKA) zu implantieren, sofern die Ligamentbalance dies zulässt.

\section{Vorausgehende Überlegungen}

Da weder die mechanische Femurachse (MF) noch die Transepikondylarachse (EKA) intraoperativ sicher zu definieren sind, war der Ausgangspunkt der Überlegung, ob man intraoperativ immer eindeutig definierbare andere Strukturen an den Femurkondylen finden kann, über die man nach entsprechenden präoperativen Voruntersuchungen die beiden wichtigen Achsen MF und EKA sicher ermitteln kann. Dabei kamen wir zu dem Ergebnis, dass die distale Femurkondylentangente geeignet ist, die mechanische Femurachse indirekt zu bestimmen, und die hintere Femurkondylentangente geeignet ist, die Transepikondylenachse auf indirektem Wege zu bestimmen, und zwar weil beide Tangenten sowohl präoperativ als auch intraoperativ eindeutig festlegbar sind.

Zur indirekten Identifikation der mechanischen Femurachse wird eine Röntgenaufnahme des gesamten Oberschenkels benötigt. Zur indirekten Identifikation der Epikondylenachse ist präoperativ ein Dünnschicht-CT erforderlich [1, $5,18]$.

Orthopäde 2014 · 43:148-155 DOI 10.1007/s00132-013-2183-0

(c) The Author(s) 2013. This article is published with open access at link.springer.com

H. Schläfer $\cdot$ M. Schläfer

\section{Achs- und rotationsgerechte Positionierung des bikondylären Femurschlittens. Präoperative Planung und intraoperative Umsetzung}

\section{Zusammenfassung}

Hintergrund. Klinisches Ergebnis und Haltbarkeit bikondylärer Oberflächenersatzprothesen sind von einer exakten Positionierung der Femurprothese abhängig. Diese sollte senkrecht zur mechanischen Femurachse und parallel zur Transepikondylarachse unter Sicherstellung einer harmonischen Ligamentbalance implantiert werden.

Methode. Weder die mechanische Femurachse noch die Transepikondylarachse sind intraoperativ ohne Hilfsmittel eindeutig definierbar. In der dargestellten Methode werden diese Achsen indirekt über die distale bzw. dorsale Femurkondylentangente bestimmt. Beide Tangenten sind sowohl präals auch intraoperativ exakt definierbar. Für die mechanische Femurachse wird präoperativ eine Röntgenaufnahme des gesamten Femurs, für die Transepikondylarachse ein Dünnschicht-CT benötigt. Die intraoperati- ve Umsetzung erfordert Steckhülsen für den 4-in-1-Block von 0 bis $13^{\circ}$ und einen speziellen Winkelmesser.

Ergebnisse. Diese Methode wurde bei 783 Patienten angewendet, 38 Patienten wurden nachuntersucht. Die Abweichung der Senkrechten zur mechanischen Femurachse betrug durchschnittlich $0,32^{\circ}$, die Abweichung von der Parallelen zur Transepikondylarachse $1,04^{\circ}$.

Schlussfolgerung. Bei der dargestellten Vorgehensweise handelt es sich um eine sichere und preiswerte Methode, die zudem die Operationszeit deutlich reduziert.

\section{Schlüsselwörter}

Bikondyläre Oberflächenersatzprothese . Rotation - Mechanische Femurachse . Hinterer Kondylenwinkel .

Transepikondylarachse

\section{Axis and rotationally correct positioning of bicondylar femoral prosthesis. Preoperative planning and intraoperative implementation}

\section{Abstract}

Background. Clinical outcome and durability of a bicondylar knee endoprosthesis depend on a correct positioning of the femoral prosthesis which should be implanted perpendicularly to the mechanical femoral axis and parallel to the transepicondylar axis to guarantee a harmonic balance of ligaments. Method. Neither the mechanical axis nor the transepicondylar axis can be correctly defined intraoperatively without an instrumental device. Using the method presented here these axes can be determined indirectly using distal and dorsal femoral condyle tangents. Both tangents can be properly defined preoperatively as well as operatively. An x-ray of the whole femur is necessary for the mechanical femoral axis and a thin-layer computed tomography (CT) scan is necessary for the transepicondylar axis. Plug-in sleeves for the 4-in- 1 block from $0-13^{\circ}$ and a special angle measurement device are required for the operative transfer.

Results. This method has been used on 783 patients of whom 38 have been examined in a follow-up study. The deviation of the perpendicular to mechanical axis was on average $0.32^{\circ}$ and the average deviation from the parallel to transepicondylar axis was $1.04^{\circ}$. Conclusion. The presented procedure is safe and economical and saves operating time.

Keywords

Bicondylar knee prosthesis - Rotation . Mechanical femoral axis · Dorsal condylar angle $\cdot$ Transepicondylar axis
Präoperative Diagnostik und intraoperative Umsetzung

\section{Indirekte Bestimmung der mechanischen Femurachse}

Präoperativ wird auf der Röntgenaufnahme des Oberschenkels die mechanische Femurachse (MF) eingezeichnet, die die
Verbindungslinie zwischen dem Hüftkopfmittelpunkt und dem Mittelpunkt des distalen Femurkondylenmassivs darstellt.

Anschließend wird bei geradem Oberschenkelknochen die anatomische Femurachse (AF) oder bei gebogenem Oberschenkelknochen eine möglichst lange ge- 

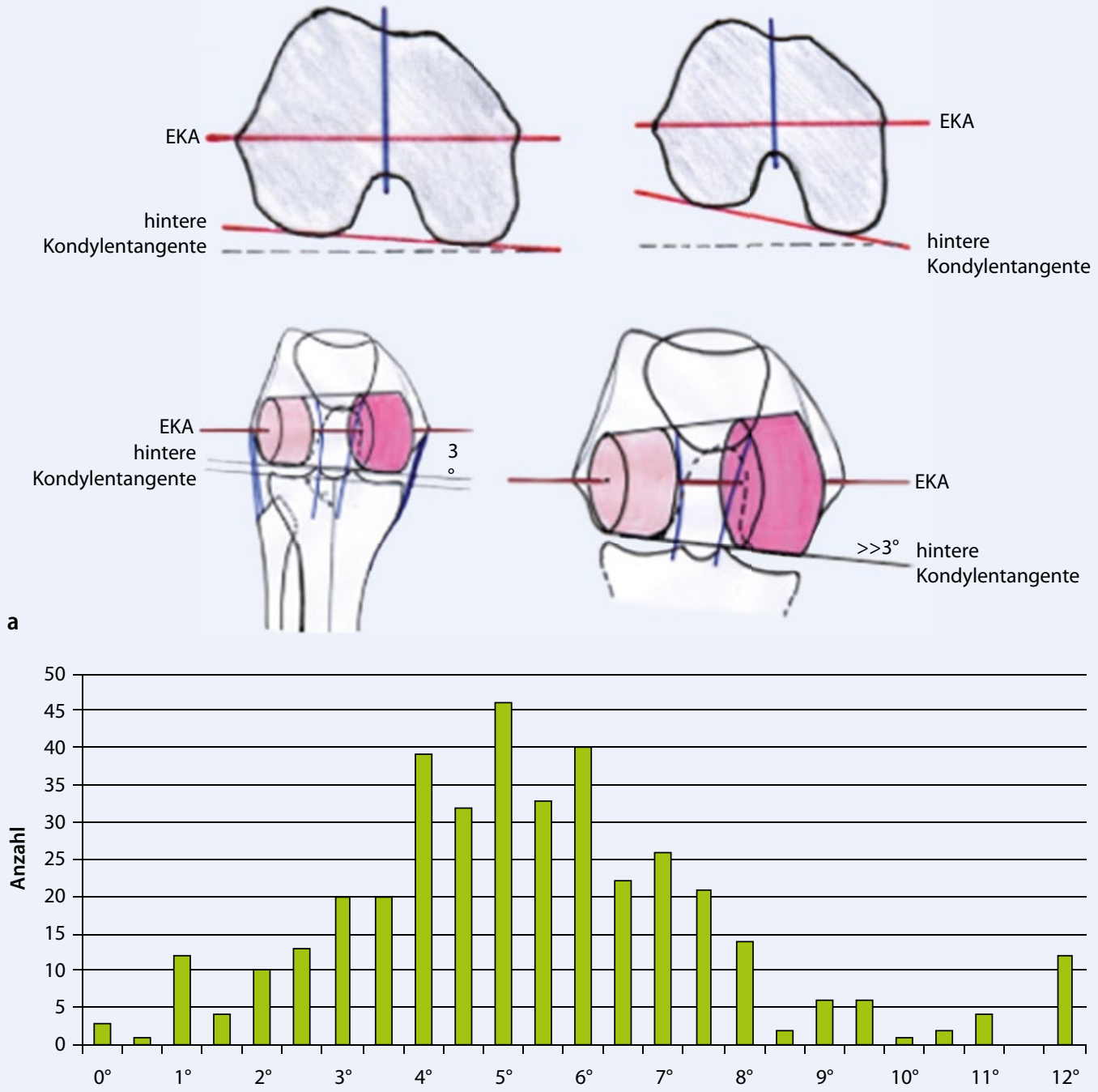

b

Hintere Kondylenwinkel
Abb. $1<$ a Schematische Darstellung unterschiedlicher anatomischer Kniegelenkformen. Bei der normoplastischen Form beträgt der hintere Kondylenwinkel ca. $3^{\circ}$, bei der dysplastischen Form $>3^{\circ}$. b Die Schwankungsbreite des hinteren Kondylenwinkels liegt zwischen 0 und $13^{\circ}$. EKA Transepikondylarachse

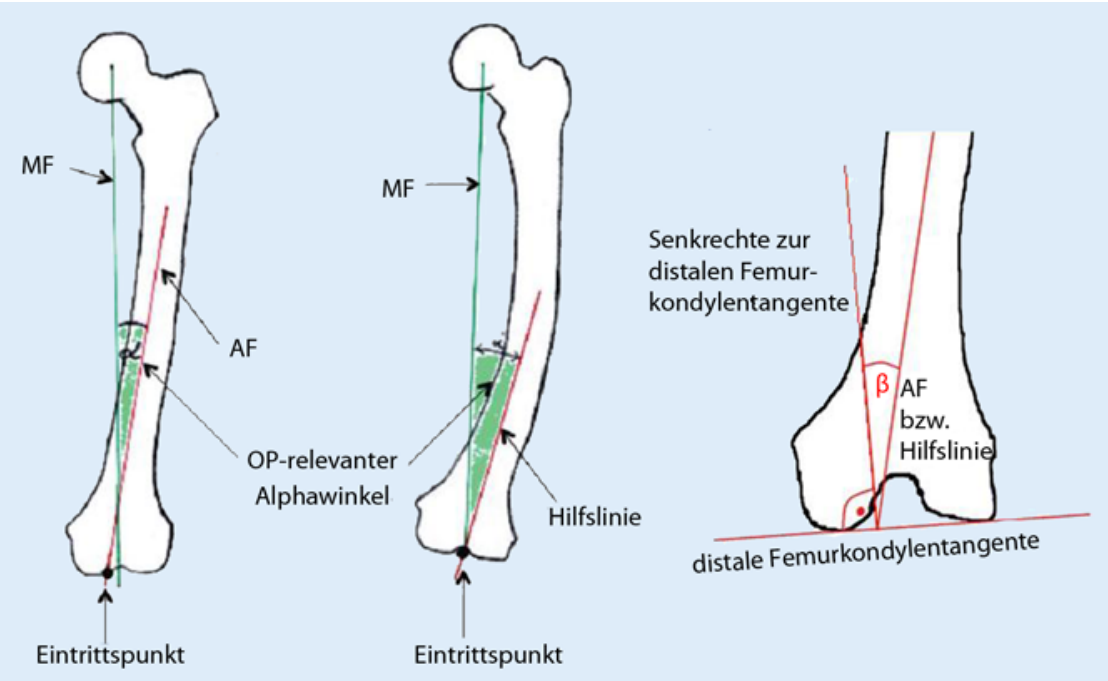

Abb. $2 \Delta$ Ermittlung des operationsrelevanten Alphawinkels und des Betawinkels bei gebogenen Oberschenkelknochen. $M F$ mechanische Femurachse, $A F$ anatomische Femurachse 


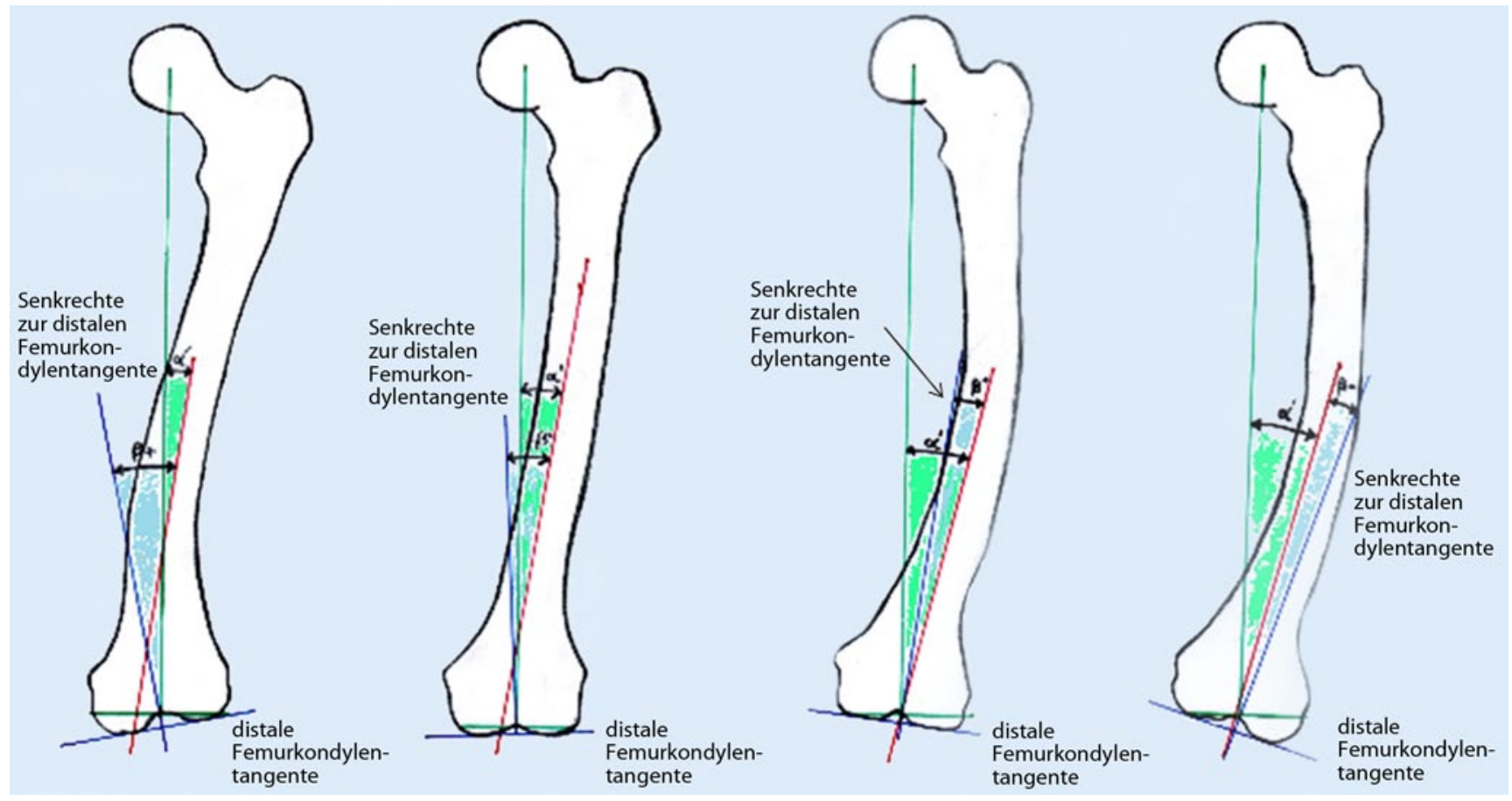

Abb. $3 \Delta$ Beispiele der Alpha- und Betawinkel bei unterschiedlich geformten Femura

rade Hilfslinie eingezeichnet. Diese dienen der späteren Lage des intraoperativ einzubringenden intramedullären Ausrichtstabs. Der distale Eintrittspunkt in den Femurmarkraum wird registriert und die Abweichung vom Mittelpunkt des Femurkondylenmassivs gemessen. Der Winkel zwischen dieser mechanischen Femurachse und der AF bzw. der Hilfslinie wird gemessen. Wir bezeichnen diesen Winkel als operationsrelevanten Alphawinkel (• Abb. 2,3).

Um die anatomische Femurachse AF bzw. die eben genannte Hilfslinie bei der Operation auch stets sicher zu treffen, messen wir noch den so genannten $\mathrm{Be}$ tawinkel: der Winkel zwischen der Senkrechten zur distalen Femurtangente und der anatomischen Femurachse bzw. der Hilfslinie (• Abb. 2, 3).

Außerdem wird jeweils die Senkrechte zur mechanischen Femurachse und zur Tibiaachse auf der Röntgenaufnahme gelenknah eingezeichnet, um eine Vorstellung von den Größenverhältnissen der zu erwartenden Knochenresektate zu erhalten.

Von jedem Operateur wird anschließend in unserer Abteilung eine präoperative Planungsskizze mit den eingezeichneten Winkeln erstellt. Das Verhältnis der Knochenresektate wird entspre- chend farblich markiert, sodass während der Operation die entnommenen Resektate mit der präoperativen Planskizze abgeglichen werden können.

Bei der Operation ist die Kenntnis folgender Marker essenziell:

- der Betawinkel (Winkel zwischen der Senkrechten zur distalen Femurkondylentangente und der AF bzw. Hilfslinie).

- der Eintrittspunkt der AF bzw. Hilfslinie in den Markraum und dessen Abweichung vom Mittelpunkt der distalen Femurkondylentangente in Millimetern.

- der operationsrelevante Alphawinkel (Winkel zwischen der mechanischen Femurachse und der AF bzw. der Hilfslinie).

Bei der Operation wird gleich zu Anfang in den 4-in-1-Sägeblock die Hülse mit dem Betawinkel eingesteckt. Nachfolgend wird der Sägeblock (4-in-1-Block) mit dem Betawinkeleinsatz an beide entknorpelten distalen Femurkondylen bündig angelegt. Der Metallblock wird soweit seitlich verschoben, bis die Steckhülse mit dem zeichnerisch ermittelten Eintrittspunkt übereinstimmt. In dieser Position wird der Markraum aufgebohrt. Man kann nun sicher sein, dass der Bohrer und der nachfolgend eingeschobene Ausrichtstab in ihrer Lage exakt der AF bzw. der Hilfslinie entsprechen (• Abb. 4).

Der Ausrichtstab bleibt im Markraum liegen. Der Metallblock wird abgezogen und die Betahülse sogleich gegen die Hülse mit dem operationsrelevanten Alphawinkel ausgetauscht und wieder auf den Ausrichtstab geschoben. Der Metallblock ist jetzt exakt senkrecht zur mechanischen Femurachse positioniert, das Ziel also erreicht.

Der distale Sägeschnitt an den Femurkondylen wird in einer späteren Operationsphase durchgeführt.

\section{Indirekte Bestimmung der Transepikondylarachse}

Nun muss nur noch die Rotation des Femurschlittens parallel zur Epikondylenachse stimmen. Um dies zu gewährleisten, lassen wir bei jedem zur bikondylären Prothesenversorgung anstehenden Patienten ein Dünnschicht-CT anfertigen.

Per Dünnschicht-CT können nämlich die Epikodylenlinie (EKL) und die hintere Kondylenlinie (HKL) sicher definiert werden [1]. Der Winkel zwischen diesen beiden Linien wird gemessen, er nennt sich hinterer Kondylenwinkel (• Abb. 5a, b; [18]). Die Schnittführung im CT verläuft hier senkrecht zur anatomischen 


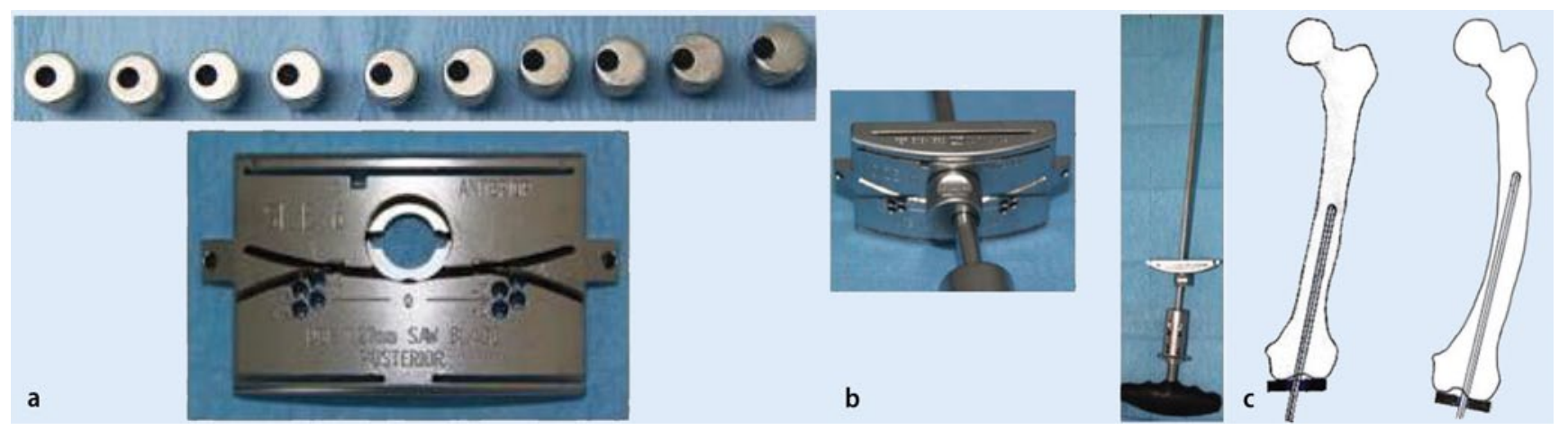

Abb. $4 \Delta$ a Steckhülsen, 4-in-1-Block. b 4-in-1-Block mit Steckhülsen und eingeschobenem intramedullärem Ausrichtstab. c 4-in-1-Block mit Betawinkelsteckhülse und eingeschobenem intramedullärem Ausrichtstab in einem geraden und einem gebogenen Femurschaft
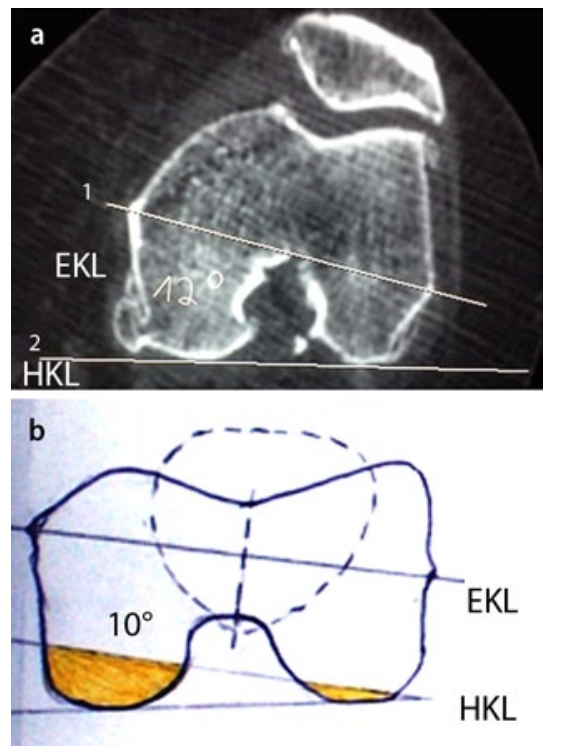

Abb. $5 \Delta$ a Ermittlung des hinteren Kondylenwinkels mittels Dünnschicht-CT. b Schematische Darstellung. EKL Epikodylenlinie, HKL hintere Kondylenlinie

Achse durch die Femurkondylen. Dieser Winkel ist für die intraoperative Bestimmung der Transepikondylarachse (EKA) von entscheidender Bedeutung. Intraoperativ wird der hintere Kondylenwinkel unter Benutzung eines speziellen Winkelmessers (• Abb. 6), der eigens für unsere Klinik konstruiert wurde, wie folgt übertragen:

Mit den Fühlern dieses Winkelmessers greifen wir die hinteren Kondylenabschnitte entsprechend der hinteren Kondylentangente $\mathrm{ab}$ (nachdem die Femurkondylen in diesem Bereich entknorpelt wurden, denn das CT gibt uns ja lediglich eine Darstellung der Knochenstrukturen bzw. -konturen). Der Winkelmesser wird dann auf den computertomographisch ermittelten hinteren Kondylenwinkel eingestellt. Somit zeigt der obere Arm des Winkelmessers ganz exakt die Verlaufsrichtung der EKA an. Das Ziel ist also erreicht. Der 4-in-1-Sägeblock wird in dieser Position mit Pins passager fixiert.

Bevor wir nun die hinteren und vorderen Sägeschnitte an den Oberschenkelrollen vornehmen, welche die Rotation des Femurschlittens festlegen, wird zunächst mit dem Rotationsspreizer überprüft, ob eine balancierte Bandspannung in Beugestellung gegeben ist. Ist dies der Fall, werden erst dann die hinteren und vorderen Sägeschnitte durchgeführt. Hier liegt der Vorteil gegenüber den MRT- und CTgestützten Operationsschablonensystemen, bei denen erst gesägt und dann die Bandspannung überprüft wird. Der Operateur wird dadurch vor vollendete Tatsachen gestellt. Meist geht das gut, aber nicht immer! In seltenen Fällen kann man bei dem Versuch, eine ausreichende Ligamentbalance erreichen zu wollen, zu einem Ligament-Overrelease gezwungen werden oder zu Nachresektionen am Knochen.

Ist bei unserer Vorgehensweise eine balancierte Bandspannung in Beugestellung nicht gegeben, werden in dieser Phase nur die Bandkapselstrukturen „released“, die für eine einseitige Einengung des Beugespalts verantwortlich sind. Gelingt über diesen Weg kein balancierter Flexionsspalt, wird zunächst ein sehr sparsamer distaler Femurcut rechtwinklig zur mechanischen Femurachse gesetzt und in Streckstellung ein balancierter Streckspalt unter permanenter Überprüfung des Flexionsspalts per Release angestrebt. Unter Umständen müssen sogar knöcherne
Kompromisse (Achsabweichung und/ oder Innenrotation bis zu $3^{\circ}$ ) eingegangen werden. Wir operieren zwar in der Tibia-first-Technik, bzgl. der Ligamentbalance hat der Streckspalt allerdings Priorität vor dem Beugespalt.

\section{Ergebnisse}

Wir konnten 38 Patienten, die entsprechend unserer Vorgehensweise von 4 Operateuren operiert wurden, nachuntersuchen. Ausgenommen wurden $\mathrm{Pa}$ tienten, bei denen ein knöcherner Kompromiss zum Erreichen eines balancierten Extensions- und Flexionsspalt eingegangen werden musste. Neben einer klinischen Untersuchung wurden zur Überprüfung unserer Vorgehensweise Routineröntgenkontrolluntersuchungen und die Durchführung eines Dünnschichtcomputertomogramms bei in der Regel bestehender femoropatellarer Symptomatik zur Überprüfung der Rotationsstellung des Femurschlittens vorgenommen.

Die Analyse der postoperativen Röntgenaufnahmen erfolgte durch 4 Untersucher, die postoperative Bestimmung der Rotationspositionierung des Femurschlittens per Dünnschicht-CT durch immer dieselbe Radiologin, die auch präoperativ den hinteren Kondylenwinkel bestimmte. Bei der klinischen Untersuchung wurden durchweg femoropatellare Probleme angegeben, was das Einschlusskriterium für die Nachuntersuchungen darstellte, um ein KontrollDünnschicht-CT zu rechtfertigen.

Das Durchschnittsalter der nachuntersuchten Patienten belief sich auf 69 
Hier steht eine Anzeige.

黛 Springer 


\section{Originalien}
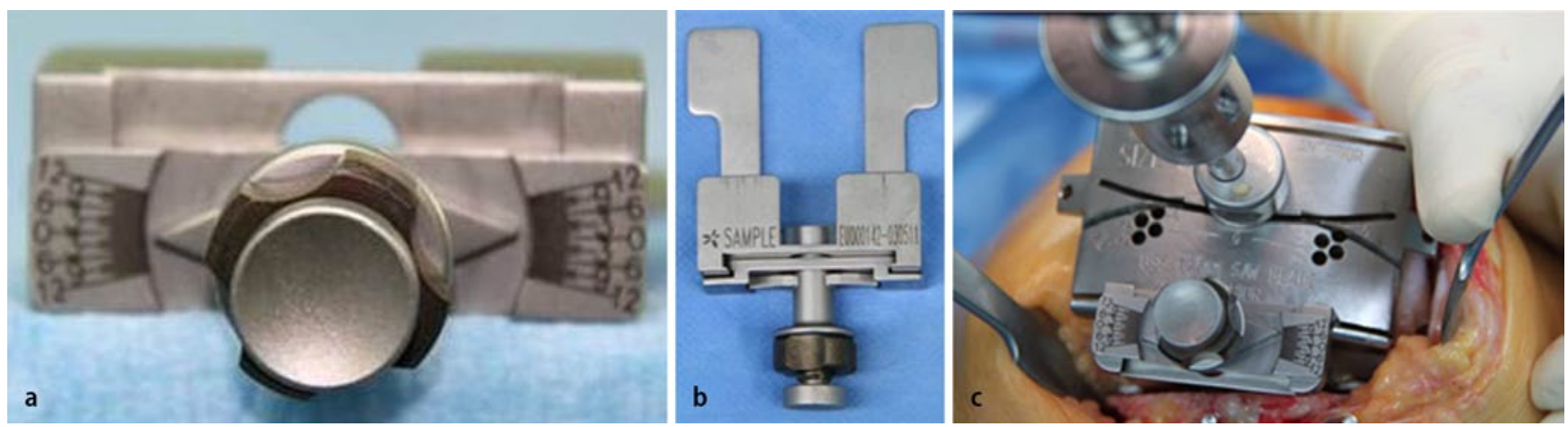

Abb. $6 \Delta$ a, b Für unsere Klinik entwickelter Winkelmesser. c Operationssitus mit Ausrichtung des 4-in-1-Blocks nach dem präoperativ ermittelten hinteren Kondylenwinkel mit dem Winkelmesser
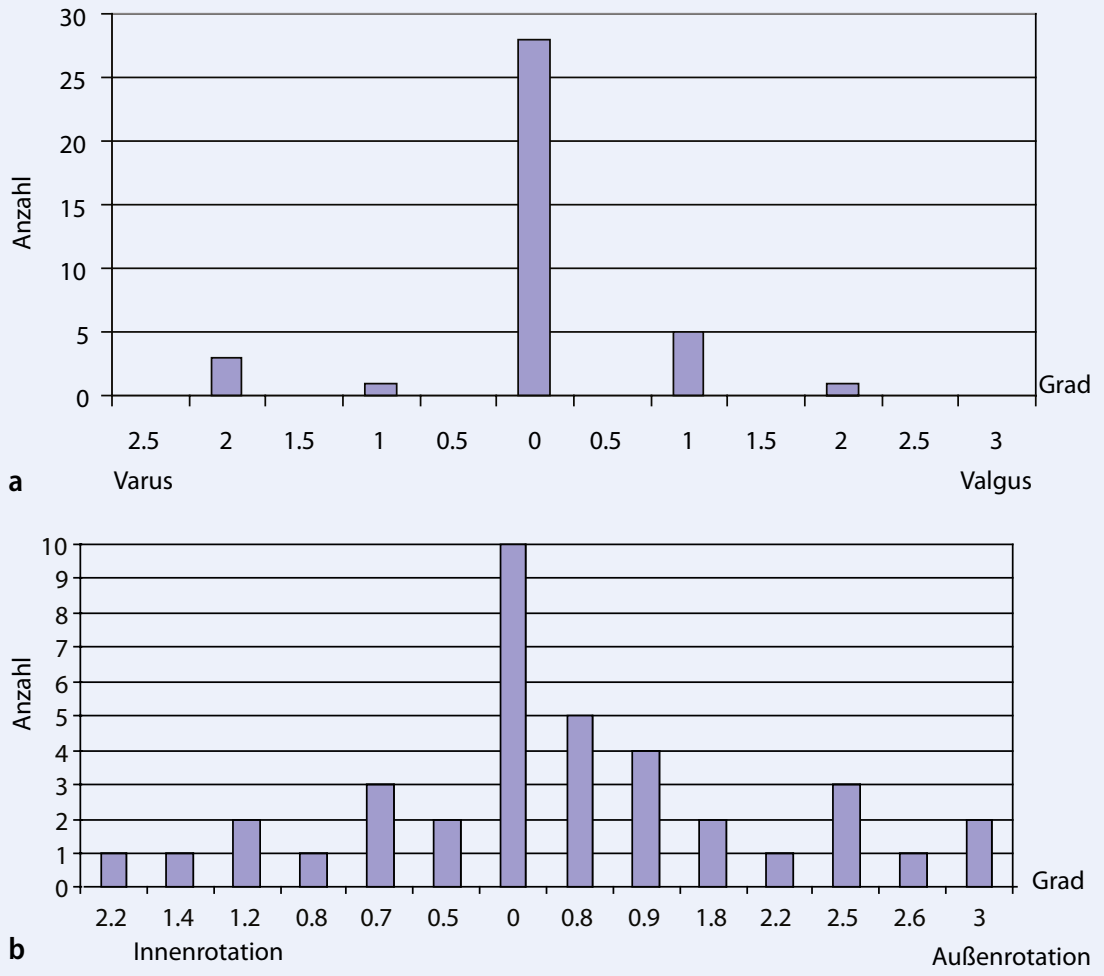

Abb. $7 \Delta$ a Gradabweichung der Femurprothese von der Senkrechten zur mechanischen Femurachse. b Gradabweichung der Femurprothese von der Transepikondylarachse

(51 bis 82 Jahre). Die Geschlechtsverteilung Männer zu Frauen lag bei 10 zu 28. Der durchschnittliche Body Mass Index (BMI) belief sich auf 28,7. Bei sämtlichen nachuntersuchten Patienten fanden sich eine gerade Beinachse, ein stabiler Bandapparat sowie eine mehr oder weniger ausgeprägte Patellalateralisation mit typischem Verschiebe- und Anpressschmerz.

Die Röntgen- und CT-Analysen führten zu folgenden Ergebnissen: die Gradabweichung der Femurprothese von der Senkrechten zur mechanischen Femur- achse lag mehrheitlich bei $0^{\circ}(\bullet$ Abb. 7a), die Gradabweichung der Femurprothese von der Transepikondylarachse (EKA) zwischen $2,2^{\circ}$ Innen- und $3^{\circ}$ Außenrotation, wobei in 10 Fällen hinsichtlich der Rotation keine Gradabweichung feststellbar war (• Abb. 7b). Die "Ausreißer“ sind u. E. entweder auf Fehler in der radiologischen Methode oder in der intraoperativen Umsetzung zurückzuführen, z. B. durch unzureichende Entknorpelung oder ungenaue Positionierung des Winkelmessers.
Auf den Patellatangentialaufnahmen kam bei 28 Patienten eine Patellalateralisation zur Darstellung. Bei der im Rahmen der Bestimmung des hinteren Kondylenwinkels per Dünnschicht-CT zusätzlich durchgeführten Torsionsanalyse des Femurs fanden sich in einem sehr hohen Prozentsatz vermehrte Endotorsionen des distalen Femurs, auf die im Rahmen dieser Arbeit nicht näher eingegangen werden kann.

Die Analyse der Operationszeiten aller 783 Prothesenoperationen ergab eine durchschnittliche Operationsdauer von 87 min, wobei die kürzeste Operationszeit $60 \mathrm{~min}$, die längste $127 \mathrm{~min}$ betrug.

\section{Diskussion}

Die seit 5 Jahren in unserer Klinik durchgeführte präoperative Planung und intraoperative Umsetzung der dargestellten Methode hat zu einer hohen Genauigkeit bzgl. der angestrebten Positionierung der bikondylären Schlittenprothese senkrecht zur MF und parallel zur EKA geführt: so beträgt die durchschnittliche Abweichung von der Senkrechten zur MF $0,32^{\circ}$, die durchschnittliche Abweichung von der EKA $1,04^{\circ}$.

Unter Berücksichtigung anzunehmender geringfügiger systembedingter Messungenauigkeiten handelt es sich unseres Erachtens um eine Vorgehensweise mit hoher Präzision. Abweichungen über den akzeptablen Toleranzbereich (Valgusoder Varusabweichung bzw. Innenrotationen der Schlittenprothese von über $3^{\circ}$ ) hinaus traten in keinem Falle auf.

Eine vollständige Eliminierung einer Patellalateralisation bzw. eines Patella- 
trackings konnte nach unseren Beobachtungen jedoch nicht in jedem Falle erzielt werden. Wir vermuten, dass in diesen Fällen eine Fehltorsion des Femurs im Sinne einer vermehrten Endotorsion des distalen Abschnitts dafür verantwortlich sein könnte. Die bei einer entsprechenden femoropatellaren Symptomatik zusätzlich durchgeführten Torsionsanalysen des Femurs bestätigen bisher unsere Vermutungen.

\section{Fazit für die Praxis}

Für die dargestellte Vorgehensweise zur angestrebten Positionierung der bikondylären Femurschlittenprothese ist mindestens eine Röntgenaufnahme des gesamten Femurs a.p. in exakter Rotation oder besser eine Ganzbeinstandaufnahme erforderlich. Ferner werden Steckhülsen für den 4-in-1-Sägeblock mit Winkeln von $0-13^{\circ}$ benötigt. Außerdem ist präoperativ per Dünnschicht-CT der hintere Kondylenwinkel, also der Winkel zwischen der hinteren Kondylentangente und der Transepikondylarachse (EKA) zu bestimmen. Für die intraoperative Übertragung dieses Winkels wird ein entsprechender Winkelmesser benötigt, behelfsweise auch ein normaler Winkelmesser. Die Femurkondylen müssen distal und dorsal entknorpelt werden, da die präoperativen Analysen sich ausschließlich am Knochen orientieren.

Die dargestellte Vorgehensweise

- stellt eine exakte landmarkenorientierte Positionierung des bikondylären Schlittens sicher,

- verkürzt die Operationszeit deutlich,

- ist preiswert,

- bedingt eine nur sehr niedrige Strahlenbelastung von $\mathrm{ca} .500 \mathrm{cGy} \times \mathrm{cm}^{2}$ (entspricht ca. der Strahlenbelastung einer Röntgenbeckenübersichtsaufnahme),

- hält die Option auf eine Ligamentbalancierung vor der knöchernen Zurichtung offen,

- reduziert femoropatellare Probleme deutlich.

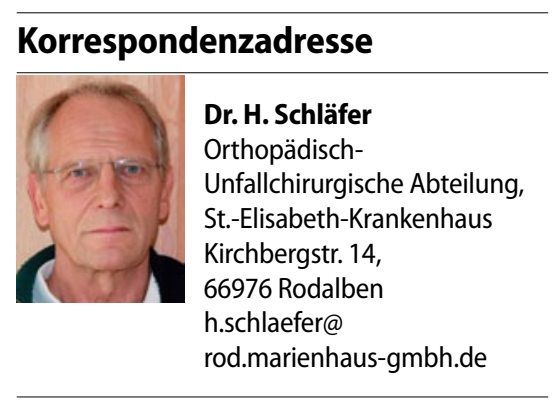

\section{Einhaltung ethischer Richtlinien}

Interessenkonflikt. H. Schläfer weist auf folgende Beziehung hin: Autorenvertrag mit der Firma Smith \& Nephew.

Dieser Beitrag beinhaltet keine Studien an Menschen oderTieren.

Open Access: This article is distributed under the terms of the Creative Commons Attribution License which permits any use, distribution, and reproduction in any medium, provided the original author(s) and source are credited.

\section{Literatur}

1. Aglietti P, Sensi L, Cuomo P, Ciardullo A (2008) Rotational position of femoral and tibial components in TKA using the femoral transepikondylar axis. Clin Orthop Relat Res 466:2751-2755

2. Akagi M, Yamashita E, Nakagawa T et al (2001) Relationship between frontal knee alignment and reference axes in the distal femur. Clin Orthop Relat Res 388:147-156

3. Anouchi YS, Whiteside LA, Kaiser AD, Milliano MT (1993) The effects of axial rotational alignment of the femoral component on knee stability and patellar tracking in total knee arthroplasty demonstrated on autopsy specimens. Clin Orthop Relat Res 287:170-177

4. Barrack RL, Schrader T, Bertot AJ et al (2001) Component rotation and anterior knee pain after total knee arthroplasty. Clin Orthop Relat Res 392:4655

5. Berger RA, Crossett LS, Jacobs JJ, Rubash HE (1998) Malrotation causing patellofemoral complications after total knee arthroplasty. Clin Orthop Relat Res 356:144-153

6. Berry DJ, Rand JA (1993) Isolated patellar component revision of total knee arthroplasty. Clin Orthop Relat Res 286:110-115

7. Buechel FF, Pappas MJ (1989) New Jersey low contact stress knee replacement system. Ten-year evaluation of meniscal bearings. Orthop Clin North Am 20:147-177

8. Churchill DL, Incavo SJ, Johnson CC, Beynnon BD (1998) The transepikondylar axis approximates the optimal flexion axis of the knee. Clin Orthop Relat Res 356:111-118

9. Griffin FM, Insall JN, Scuderi GR (2000) Accuracy of soft tissue balancing in total knee arthroplasty. J Arthroplasty 15:970-973
10. Hofmann S, Romero J, Roth-Schiffl E, Albrecht T (2003) Rotational malalignment of the components may cause chronic pain or early failure in total knee arthroplasty. Orthopade 32:469-476

11. Insall JN, Scuderi GR, Komistek RD et al (2002) Correlation between kondylar lift-off and femoral component alignment. Clin Orthop Relat Res 120:143-152

12. Jerosch J, Peuker E, Philipps B, Filler T (2002) Interindividual reproducibility in perioperative rotational alignment of femoral components in knee prosthetic surgery using the transepikondylar axis. Knee Surg Sports Traumatol Arthrosc 10:194-197 (official journal of the ESSKA)

13. Katz MA, Beck TD, Silber JS et al (2001) Determining femoral rotational alignment in total knee arthroplasty: reliability of techniques. J Arthroplasty 16:301-305

14. Miller MC, Berger RA, Petrella AJ et al (2001) Optimizing femoral component rotation in total knee arthroplasty. Clin Orthop Relat Res 392:38-45

15. Olcott CW, Scott RD (1999) The Ranawat Award. Femoral component rotation during total knee arthroplasty. Clin Orthop Relat Res 367:39-42

16. Romero J, Duronio JF, Sohrabi A et al (2002) Varus and valgus flexion laxity of total knee alignment methods in loaded cadaveric knees. Clin Orthop Relat Res 294:243-253

17. Romero J, Stahelin T, Binkert C et al (2007) The clinical consequences of flexion gap asymmetry in total knee arthroplasty. J Arthroplasty 22:235-240

18. Tomczak RJ, Guenther KP, Rieber A et al (1997) MR imaging measurement of the femoral antetorsional angle as a new technique: comparison with CT in children and adults. AJR Am J Roentgenol 168:791-794

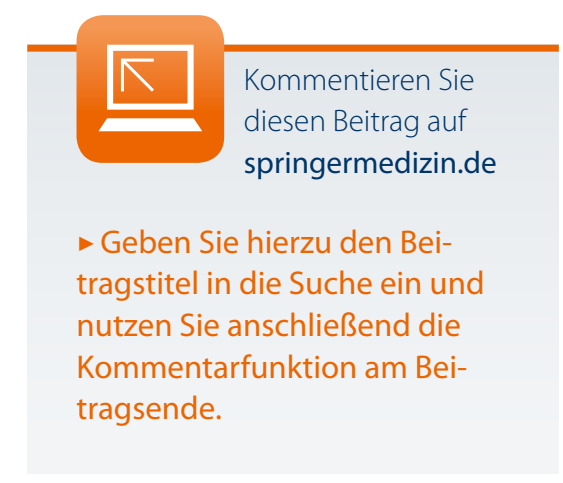

